\title{
Quantum effects in photosensitization: The case of singlet oxygen generation by thiothymines
}

\author{
Meghna A. Manae* and Anirban Hazra \\ Department of Chemistry, Indian Institute of Science Education and Research Pune, \\ Dr. Homi Bhabha Road, Pune 411008, Maharashtra, India
}

\begin{abstract}
Photosensitization is the indirect electronic excitation of a molecule with the aid of a photosensitizer and is a bimolecular nonradiative energy transfer. In this study, we have attempted to elucidate its mechanism, and we do this by calculating rate constants of photosensitization of oxygen by thiothymines (2-thiothymine, 4-thiothymine and 2,4 dithiothymine). The rate constants have been calculated using two approaches: (a) a classical limit of Fermi's Golden Rule (FGR), and (b) a time-dependent variant of FGR, where the treatment is purely quantum mechanical. The former approach has previously been developed for bimolecular systems and has been applied to the photosensitization reactions studied here. The latter approach, however, has so far only been used for unimolecular reactions, and in this work, we describe how it can be adapted for bimolecular reactions. Experimentally, all three thiothymines are known to have significant singlet oxygen yields, which are indicative of similar rates. Rate constants calculated using the time-dependent variant of FGR are comparable across all three thiothymines and with experiment. While the classical approximation gives reasonable rate constants for 2-thiothymine, it severely underestimates them for 4-thiothymine and 2,4 dithiothymine, by several orders of magnitude. This work indicates the importance of quantum effects in driving photosensitization.
\end{abstract}

\footnotetext{
* meghna @jncasr.ac.in; Present address: Theoretical Sciences Unit, Jawaharlal Nehru Centre for Advanced Scientific Research, Bangalore 560064, Karnataka, India
} 


\section{INTRODUCTION}

Photosensitization is a light mediated physical reaction, where the molecule irradiated with light is not the final target of electronic excitation. Here, a photosensitizer (PS), is photoexcited and then transfers its excitation energy to the target molecule, leading to its excitation, while the PS returns to its ground state. A common target of photosensitization is the oxygen molecule since

direct photoexcitation from its ground triplet state, ${ }^{3} \mathrm{O}_{2}$, to its singlet state, ${ }^{1} \mathrm{O}_{2}$, is spin-forbidden, and this reaction (Eq. 1) is the focus of this study.

$$
{ }^{3} \mathrm{PS}+{ }^{3} \mathrm{O}_{2} \rightarrow{ }^{1} \mathrm{PS}+{ }^{1} \mathrm{O}_{2}
$$

Photosensitization of $\mathrm{O}_{2}$ is widely made use of in organic synthesis [1-5], photovoltaics [6], and is also a key step in photodynamic therapy [7-9]. While photosensitization has been the subject of several theoretical studies [10-17], a detailed mechanistic understanding of the process is still lacking. Our aim, in this study, is to better understand photosensitization, using thiothymines as the photosensitizers, since all three thiothymines (shown in Fig. 1) - 2-thiothymine (2tThy), 4-thiothymine (4tThy) and 2,4-dithiothymine (dtThy) - are known to have significant singlet oxygen yields [18]. Moreover, the thiothymines are known to have ultrafast intersystem crossing (ISC) lifetimes [18] and near unity triplet yields with long long triplet lifetimes, of the order of microseconds $[19,20]$, making them excellent photosensitizers. Specifically, we wish to calculate the rate constant at which they photosensitize $\mathrm{O}_{2}$, and thereby obtain physical and mechanistic insights into photosensitization.

Generally, photosensitization is a nonradiative bimolecular energy transfer reaction (Eq. 1). While the spins of the individual components change, if the composite system, PS- $\mathrm{O}_{2}$, is considered, its spin multiplicity remains singlet (Eq. 2). Therefore, photosensitization can be thought of as internal conversion (IC) $[15,16]$. The critical difference from IC, however, is that while conical intersections, which mediate IC, are characterized by large couplings between the electronic states, the couplings between the electronic states in PS- $\mathrm{O}_{2}$ are relatively low. Therefore, Fermi's Golden Rule (FGR), derived using time-dependent perturbation theory, can be used to calculate the rate constant of photosensitization [21, 22].

$$
\left({ }^{3} \mathrm{PS}+{ }^{3} \mathrm{O}_{2}\right)^{1} \rightarrow\left({ }^{1} \mathrm{PS}+{ }^{1} \mathrm{O}_{2}\right)^{1}
$$




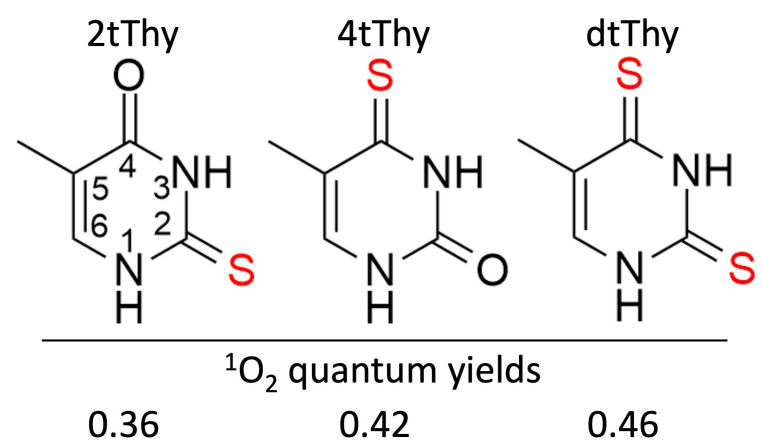

FIG. 1. The three thiothymines, 2-thiothymine (2tThy), 4-thiothymine (4tThy), and 2,4-dithiothymine (dtThy), with 2tThy showing the numbering scheme in the thiothymines. The numbers shown below are the respective singlet oxygen quantum yields of the molecules, taken from Ref. [18]

A simple means to model photosensitization, without considering potential energy surfaces, involves looking at the thermodynamics of the entities in Eq. 1 [10-13]. Without commenting on mechanistic insights or kinetics, this approach can help determine if the process is thermodynamically favourable. Dumont et. al. have considered the structures of the reactants and products of Eq. 1 and have calculated the energy along the interpolated path between the two [14], thus, obtaining an approximate potential energy surface of photosensitization. Going one step further, Serrano-Andres and co-workers have calculated the photosensitization rate constants of molecules in the furocoumarin family using FGR [15]. They calculate the coupling in a perturbative manner, and use the value of density of states explicitly computed in earlier studies [23]. More recently, Bai and Barbatti, have developed the Divide-to-Conquer method, a classical approach to calculate photosensitization rate constants [16]. This method was used to estimate rate constants in 6-aza-2-thiothymine and found to match well with experimental values [17].

As stated above, owing to photosensitization being a weak-coupling mediated reaction, FGR $[21,22]$ can be used, which gives the rate constant of nonradiative transition between an initial state to a continuum of final states, due to a weak perturbation, and is given as

$$
k_{I F}=\frac{2 \pi}{\hbar} \sum_{f}\left|\left\langle\Psi_{I i}|\hat{H}| \Psi_{F f}\right\rangle\right|^{2} \delta\left(E_{I i}-E_{F f}\right)
$$

where, $\hat{H}$ couples the initial and final states $\Psi_{I i}$ and $\Psi_{F f}$ with energies $\left(E_{I i}\right)$ and $\left(E_{F f}\right)$, respectively. The uppercase and lowercase variables are used to label electronic and vibrational states, respectively. The Dirac delta function in Eq. 3 ensures conservation of energy. Within the BornOppenheimer approximation, $\Psi_{N n}=\psi_{N} \phi_{n}$, and since the coupling to a good approximation does 


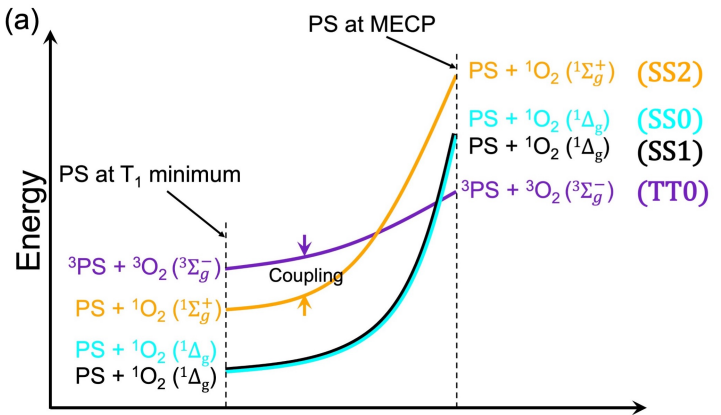

Internal coordinates of the PS (b)

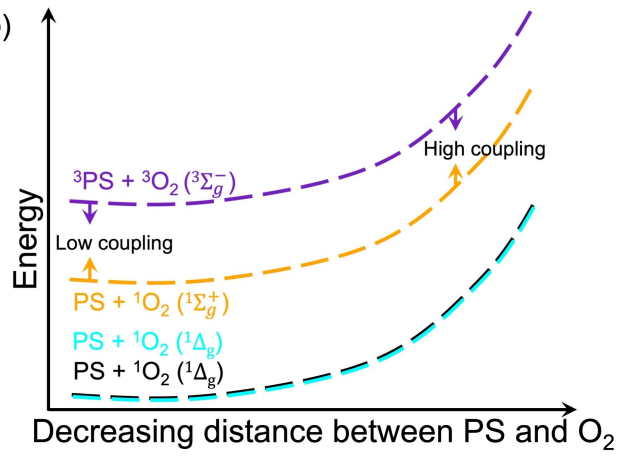

FIG. 2. Schematic showing the ordering of first four singlet states for the PS-O${ }_{2}$ composite system in (a) Rspace, at two significant geometries of the PS: $\mathrm{T}_{1}$ minimum and minimum energy crossing point (MECP), and in (b) D-space. Note that in R-space, value of D is kept large, and in D-space, the internal coordinates of the PS is kept at its $\mathrm{T}_{1}$ minimum.

not depend on the vibrational part of the wave function Eq. 3 becomes,

$$
\begin{aligned}
k_{I F} & =\frac{2 \pi}{\hbar}\left|V_{I F}\right|^{2} \mathrm{FCWD} \\
\mathrm{FCWD} & =\sum_{f}\left|\left\langle\phi_{i} \mid \phi_{f}\right\rangle\right|^{2} \delta\left(\Delta E_{I F}+E_{i}-E_{f}\right)
\end{aligned}
$$

where, $\left|V_{I F}\right|^{2}=\left|\left\langle\psi_{I}|\hat{V}| \psi_{F}\right\rangle\right|^{2}$ is the square of the diabatic coupling, $\Delta E_{I F}=E_{I}-E_{F}$ is the electronic energy gap, and $\left|\left\langle\phi_{i} \mid \phi_{f}\right\rangle\right|^{2}$, is the square of the overlaps of the initial and final vibrational wavefunctions and are called Franck-Condon Factors (FCF). The FCFs along with the delta function is called Franck-Condon weighted density of states or FCWD. Handling the delta function in FCWD poses a computational difficulty. This can be handled by either taking a classical approximation of FGR [16, 24], or by using the time-dependent variant of FGR [25-27].

Taking the classical approximation of FGR greatly simplifies FCWD and Eq. 4 reduces to the Marcus expression [24],

$$
k_{I F}^{M T}=\frac{2 \pi}{\hbar}\left|V_{I F}\right|^{2} \frac{1}{\sqrt{4 \pi \lambda k_{B} T}} e^{\frac{-\Delta G^{\ddagger}}{k_{B} T}}
$$

where, $\lambda$ is the reorganization energy and $\Delta G^{\ddagger}$ is the free activation energy of the reaction. By neglecting entropy contributions, $\Delta G^{\ddagger}$ can be replaced with the activation energy, $\Delta E^{\ddagger}$. In this work, this approach is abbreviated as Marcus Theory FGR (MT-FGR).

Implementation of MT-FGR (Eq. 5) for bimolecular energy transfer was proposed by Bai and Barbatti who suggested dividing the space of the PS- $\mathrm{O}_{2}$ system into an R-space where the internal 
coordinates of PS change, and a D-space where the distance between the PS and $\mathrm{O}_{2}$ is varied (Fig. 2) [16]. This separation is made on the basis of the strong covalent interactions within the PS having a different role in the reaction as compared to the weak van der Waals interaction between the PS and $\mathrm{O}_{2}$. The ordering of the first four singlet states of the PS-O $\mathrm{O}_{2}$ at the $\mathrm{T}_{1}$ minimum geometry of the PS (Fig. 2a) is dominated by the energetics of the PS, since its absolute electronic energy is significantly larger in magnitude compared to $\mathrm{O}_{2}$. Therefore, despite the geometry being the $\mathrm{T}_{1}$ minimum, its $\mathrm{S}_{0}$ energy will be lower than its $\mathrm{T}_{1}$ energy. At the minimum energy crossing point (MECP) geometry of the PS, however, the $\mathrm{S}_{0}$ and $\mathrm{T}_{1}$ states are degenerate and the ordering of the first four singlet states will be dominated by $\mathrm{O}_{2}$, and thus, the ground triplet state of $\mathrm{O}_{2}$ will be lowest in energy. Since, the ordering of the first four singlet states for the PS- $\mathrm{O}_{2}$ system in R-space are different at the $\mathrm{T}_{1}$ minimum and MECP geometries of the PS (Fig. 2a), this mode promotes a crossing between the reactant $\left({ }^{3} \mathrm{PS}-{ }^{3} \mathrm{O}_{2}\left({ }^{3} \Sigma_{g}^{-}\right)\right)$and the product states (doubly degenerate ${ }^{1} \mathrm{PS}-$ ${ }^{1} \mathrm{O}_{2}\left({ }^{1} \Delta_{g}\right)$ and $\left.{ }^{1} \mathrm{PS}-{ }^{1} \mathrm{O}_{2}\left({ }^{1} \Sigma_{g}^{+}\right)\right)$. In the present study, these states are labelled TT0, SS0, SS1, and SS2, respectively, and we only focus on the transition from the TT0 to SS2 state. The diabatic coupling is assumed to be independent of changes in the internal coordinates of the PS and only depends on the distance between the PS and $\mathrm{O}_{2}$ and is hence calculated as a function of changing coordinates in the D-space (Fig. 2b).

For the time-dependent variant of FGR, using $\delta\left(\Delta E_{I F}+E_{i}-E_{f}\right)=\frac{1}{2 \pi} \int_{-\infty}^{\infty} e^{i \tau\left(\Delta E_{I F}+E_{i}-E_{f}\right)} d \tau$, the following form, in atomic units, is obtained,

$$
\begin{aligned}
k_{I F} & =\left|V_{I F}\right|^{2} \int_{-\infty}^{\infty} G(\tau) e^{i \tau\left(\Delta E_{I F}+E_{i}\right)} d \tau \\
G(\tau) & =\sum_{f}\left|\left\langle\phi_{i} \mid \phi_{f}\right\rangle\right|^{2} e^{-i \tau E_{f}}
\end{aligned}
$$

Here, $G(\tau)$ is the generating function for the vibrational wavefunctions, which within the harmonic approximation are the eigenfunctions of the harmonic oscillator. Since this approach transforms a function of energy to a function of time, it is termed the time-dependent variant of FGR (TD-FGR) [25].

In this work, we calculate and compare the photosensitization rate constants obtained from MTFGR and TD-FGR. We find that the rate constants from MT-FGR (classical approximation) are severely underestimated as compared to experimental values, but the rate constants from TD-FGR are in reasonable agreement with experiment. Our results point to the significance of quantum effects in photosensitization.

In the subsequent section, we describe how TD-FGR can be adapted to model a bimolecular 
reaction. This is followed by the computational methods used and the details of our calculations. The rate constants of photosensitization are then presented for the three thiothymines using both MT-FGR and TD-FGR, followed by a discussion on how the two approaches fare. Finally, the conclusions of our study are presented.

\section{ADAPTING TD-FGR FOR BIMOLECULAR ENERGY TRANSFER}

TD-FGR has been used to calculate rate constants in quantum processes such as ISC [25, 28] and IC [29-32] and calculated values are known to be in agreement with experiment. However, it has only been applied for unimolecular reactions so far. Despite considering photosensitization as IC of the PS- $\mathrm{O}_{2}$ composite system, there are several complexities that arise since PS- $\mathrm{O}_{2}$ is, in fact, a bimolecular system. In this section, we highlight these intricacies and how to tackle them.

\section{A. Duschinsky relation and imaginary modes}

The infinite sum in $N$ dimensions in Eq. 6, $G(\tau)$, can be reduced to a closed-form expression using Mehler's formula [33].

$$
\begin{array}{r}
G(\tau)=(\sqrt{2} \pi)^{-N}\left(\operatorname{det}\left(\mathbf{S}^{-1} \Omega_{I} \Omega_{F}\right)\right)^{\frac{1}{2}} \times \\
\int e^{-\frac{1}{4}\left(\left(\mathbf{Q}_{F}+\overline{\mathbf{Q}}_{F}\right)^{\dagger} \Omega_{F} \mathbf{B}\left(\mathbf{Q}_{F}+\overline{\mathbf{Q}}_{F}\right)\right)} \times \\
e^{-\frac{1}{4}\left(\left(\mathbf{Q}_{F}-\overline{\mathbf{Q}}_{F}\right)^{\dagger} \Omega_{F} \mathbf{B}^{-1}\left(\mathbf{Q}_{F}-\overline{\mathbf{Q}}_{F}\right)+2 \mathbf{Q}_{I}^{\dagger} \Omega_{I} \mathbf{Q}_{I}+2 \overline{\mathbf{Q}}_{I}^{\dagger} \Omega_{I} \overline{\mathbf{Q}}_{I}\right)} d \mathbf{Q}_{I} d \overline{\mathbf{Q}}_{I}
\end{array}
$$

Here, $\Omega_{I}$ is a diagonal matrix with elements $\Omega_{i i}=\omega_{i}$, and $\omega_{i}$ corresponds to the frequencies of the initial state. $\Omega_{F}, \mathbf{S}$ and $\mathbf{B}$ are diagonal matrices with elements $\Omega_{i i}=\omega_{i}, S_{i i}=\sinh \left(i \omega_{i} \tau\right)$ and $B_{i i}=\tanh \left(i \omega_{i} \tau / 2\right)$, respectively, and $\omega_{i}$ corresponds to the frequencies of the final state. $\mathbf{Q}_{I}$ and $\mathbf{Q}_{F}$ are column vectors composed of the mass-weighted normal mode coordinates of the initial and final states, respectively. The integration in Eq. 7 is over the two sets of mass weighted normal mode coordinates (one barred and one unbarred) of the initial state. The normal coordinates of the final states, $\mathbf{Q}_{F}$, are related to the normal coordinates of the initial state, $\mathbf{Q}_{I}$ by a Duschinsky transformation [34]:

$$
\mathbf{Q}_{F}=\mathbf{J} \mathbf{Q}_{I}+\mathbf{D}
$$


where, $\mathbf{J}=\mathbf{Q}_{F}^{\dagger} \mathbf{Q}_{I}$, is the Duschinsky rotation matrix, and $\mathbf{D}=\mathbf{Q}_{F}^{\dagger}\left(\mathbf{R}_{I}-\mathbf{R}_{F}\right)$, is the displacement vector. Including Duschinsky effects accounts for changes in normal coordinates of different electronic states. We have also ensured that the vibrational displacements satisfy the Eckart conditions $[35,36]$.

Using the Duschinsky transformation, Eq. 7 reduces to,

$$
\begin{array}{r}
G(\tau)=2^{\frac{N}{2}} \sqrt{\frac{\operatorname{det}\left(\mathbf{S}^{-1} \Omega_{I} \Omega_{F}\right)}{\operatorname{det}\left(\mathbf{J}^{\dagger} \Omega_{F} \mathbf{B J}+\Omega_{I}\right) \operatorname{det}\left(\mathbf{J}^{\dagger} \Omega_{F} \mathbf{B}^{-1} \mathbf{J}+\Omega_{I}\right)}} \\
\times e^{\mathbf{D}^{\dagger}\left(\Omega_{F} \mathbf{B} \mathbf{J}\left(\mathbf{J}^{\dagger} \Omega_{F} \mathbf{B J}+\Omega_{I}\right)^{-1} \mathbf{J}^{\dagger} \Omega_{F} \mathbf{B}-\Omega_{F} \mathbf{B}\right) \mathbf{D}}
\end{array}
$$

Finally, the photosensitization rate constant, $k_{I F}^{T D}$, is calculated using (Eq. 9), the origin of which is explained below.

$$
k_{I F}^{T D}=e^{\Delta E_{i} \beta} k_{I F}=e^{\Delta E_{i} \beta}\left|V_{I F}\right|^{2} \int_{-\infty}^{\infty} G(\tau) e^{i \tau\left(\Delta E_{I F}+E_{i}\right)} d \tau
$$

The rate constant, $k_{I F}$ in Eq. 6, being quantum mechanical, considers the microscopic transition rate. For a macroscopic equivalent of this rate, a thermally equilibrated manifold of the initial vibrational states, $\{i\}$, needs to be considered [24, 25]. Therefore, the rate constant $k_{I F}^{T D}$ in Eq. 9, is $k_{I F}$ given in Eq. 6, along with a Boltzmann factor, where $\beta=1 / k_{B} T$ and $\Delta E_{i}$ is the energy of the vibrational state of TT0 electronic state with respect to its ground vibrational state. A more detailed derivation to obtain Eqs. 7, 8 and 9 from Eq. 6 can be found in the supporting information of Reference [25].

To implement Eq. 8, we need to calculate optimized geometries and normal modes of PS$\mathrm{O}_{2}$ on TT0 and SS2 states for various D values. Since these states are repulsive with respect to decreasing D (Fig. 2b), we need to perform constrained optimization of the PS- $\mathrm{O}_{2}$ complexes, with $\mathrm{D}$ as the constraint, to prevent the PS and $\mathrm{O}_{2}$ from moving apart. This sometimes leads to imaginary eigenvalues along modes that have not been optimized. While a simple solution might be to eliminate these modes, the number of such imaginary modes for the TT0 and SS2 states are unequal. To avoid the mathematical difficulty associated with imaginary frequencies, which correspond to unbound potentials, we have replaced them with a low value of $100 \mathrm{~cm}^{-1}$.

\section{B. Damping factor}

A time integration of the complex function in Eq. 9, needs to be carried out to calculate FCWD. Conveniently, the imaginary part of the complex function is an odd function and therefore its 
integral is zero. Further, the real part is an even function, and its integral can be taken as twice the value of the integral over positive time. However, the time interval over which the integration needs to be performed also has to be determined. The real part of the function over positive time is an oscillating function (Fig. S1a) and we find that the function does not get damped even after 3 ps. The inset of Fig. S1a, however, suggests that the bulk of the area under the curve is within the first few oscillations and integration over the rest of the function would cancel each other out. In this case, the value of the integral should start converging after some $\tau$. However, we see from Table S1 that the value does not converge for different time intervals.

Therefore, we have chosen to damp $\operatorname{FCWD}(\tau)$ using a Gaussian damping function [25]. This damping factor is chosen such that the function gets damped between 100 and $200 \mathrm{fs}$. The reasoning here is that the rate calculation is carried out for a certain configuration of $\mathrm{O}_{2}$ and the PS, at a particular D. However, since the timescale of nuclear motion is a few fs, the $\mathrm{O}_{2}$ is likely to change its position with respect to the PS. Essentially, 100-200 fs is all the time that the PS has to transfer its energy to the $\mathrm{O}_{2}$ molecule. Moreover, the damping factor also accounts for any radiative damping that the system may undergo, and damping due to the solvent [25]. We also see that the rate constants do not change significantly on using various damping parameters as seen in Table S2. Based on this, we have used a damping parameter of $0.1 \mathrm{~cm}^{-1}$ for all the thiothymines and carried out the time integration till $200 \mathrm{fs}$, by which time, the function most certainly gets damped (Fig. S1b) [25].

\section{Diabatic coupling and orientation directions}

Diabatic couplings can be calculated from various properties including nonadiabatic coupling, density matrix, and dipole or quadrupole moments. Here, we calculate the diabatic coupling starting with the nonadiabatic coupling between only the two states of interest (TT0 and SS2). The diabatic states are obtained by a unitary transformation of the adiabatic states such that the trans-

formation minimizes the nonadiabatic coupling projected along $\vec{D}$, which is the vector between the PS and $\mathrm{O}_{2}[16,37]$. Therefore, the diabatic coupling strongly depends on the orientation of the PS with respect to $\mathrm{O}_{2}$ as the two approach each other. Typically, the diabatic calculation would have to be performed for several orientation directions [17]. Serrano-Andres and coworkers showed that diabatic coupling is maximized with orbital overlap.[15] Further, the nature of the lowest triplet state in all 3 thiothymines was found to be $\pi \pi^{*}$ [38]. Therefore, we have chosen three approach 

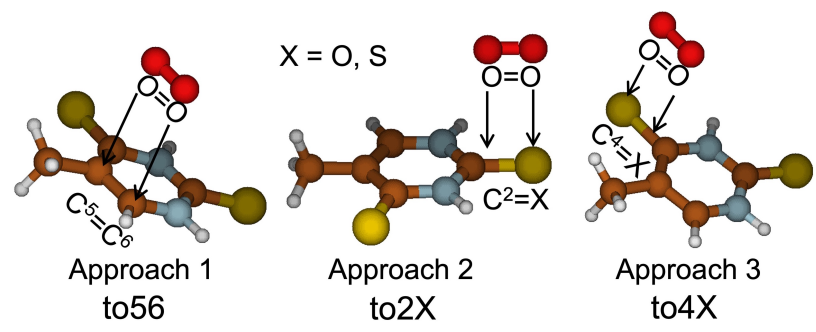

FIG. 3. The 3 approach directions along which the diabatic couplings and rate constants are calculated in this study.

directions (shown in Fig. 3), labelled as 'to56' (when $\mathrm{O}_{2}$ approaches the $\mathrm{C}^{5}$ and $\mathrm{C}^{6}$ double bond in the PS), 'to $2 \mathrm{X}$ ' and 'to $4 \mathrm{X}$ ' (when $\mathrm{O}_{2}$ approaches the carbonyl or thione at the 2nd and 4th positions, respectively). In all 3 approaches, the $\mathrm{O}_{2}$ is kept parallel to the PS, as this maximizes orbital overlap [15].

To obtain the diabatic coupling, the nonadiabatic coupling is projected along $\vec{D}_{t o 56}, \vec{D}_{t o 2 X}$ and $\vec{D}_{t o 4 X}$ vectors for the to56, to2X and to4X directions, respectively, which are given by Eqs. 10

$$
\begin{aligned}
\vec{D}_{t o 56} & =\frac{\vec{Q}_{C 5}^{P S}+\vec{Q}_{C 6}^{P S}}{2}-\frac{\vec{Q}_{O 1}+\vec{Q}_{O 2}}{2} \\
\vec{D}_{t o 2 X} & =\frac{\vec{Q}_{C 2}^{P S}+\vec{Q}_{X 2}^{P S}}{2}-\frac{\vec{Q}_{O 1}+\vec{Q}_{O 2}}{2} \\
\vec{D}_{t o 4 X} & =\frac{\vec{Q}_{C 4}^{P S}+\vec{Q}_{X 4}^{P S}}{2}-\frac{\vec{Q}_{O 1}+\vec{Q}_{O 2}}{2}
\end{aligned}
$$

where, $\vec{Q}^{P S}$ and $\vec{Q}_{O}$ denote the position vector of the atoms in the PS and $\mathrm{O}_{2}$, respectively. The initial magnitude of $\vec{D}$ is taken to be $3.8 \AA$, since the PS- $\mathrm{O}_{2}$ energy was found to be the sum of the energies of the PS and $\mathrm{O}_{2}$ at this distance, implying zero to negligible coupling. The final D considered was $2.2 \AA$, because by this distance, the energy of the TT0 state increases to such an extent that the rate constant is all but extinguished due to the Boltzmann factor, $e^{\Delta E_{i} \beta}$, in Eq. 9 .

Here, we make the assumption that the diabatic coupling is only dependent on the D coordinate and mostly independent of the internal coordinates of the PS, since the nature of the states do not change significantly for a fixed D. This assumption is evaluated by comparing the calculated couplings with those obtained by a more direct, although cumbersome, method. This is done by changing the internal coordinates (keeping the D fixed) till the crossing is reached between the TT0 and SS2 states. The diabatic coupling is then calculated as half the energy gap between the states at the crossing geometry [37]. Such a crossing is seen in Fig. S2 for the $T_{1}^{\text {ring }}$ conformer of 4tThy along the to $4 \mathrm{X}$ approach direction, with the D fixed at $2.5 \AA$. The diabatic coupling here (inset in 
Fig. S2) is found to be $58.2 \mathrm{~cm}^{-1}$ while the calculated diabatic coupling between these states is $27.6 \mathrm{~cm}^{-1}$. Such a comparison was done for the other two thiothymines as well: for $2 \mathrm{tThy}$ (along to56 direction with a D of $2.8 \AA$ ) and dtThy (along to $4 \mathrm{X}$ direction with a D of $2.4 \AA$ ), and the diabatic coupling was calculated to be $6.6 \mathrm{~cm}^{-1}$ and $189.5 \mathrm{~cm}^{-1}$, respectively, while that obtained by changing their internal coordinates was found to be $1.0 \mathrm{~cm}^{-1}$ and $115.9 \mathrm{~cm}^{-1}$, respectively. In all three cases, the diabatic coupling obtained with two different methods are similar and of the same order of magnitude.

\section{COMPUTATIONAL METHODOLOGY}

Multi-reference electronic structure methods are necessary due to the open-shell characters of PS and $\mathrm{O}_{2}$ in the photosensitization reaction. In this work, geometry optimizations, frequency, nonadiabatic couplings and single-point energy calculations of the composite PS- $\mathrm{O}_{2}$ system were carried out at the SA-CASSCF [39, 40] level of theory with a state-averaging that included the first 4 singlet states. The active space consisted of 10 electrons in 8 orbitals, denoted as 10,8, of which 6,4 belonged to the $\mathrm{O}_{2}$ and 4,4 belonged to the PS. The orbitals for $\mathrm{O}_{2}$ included its $2 \pi$ and $2 \pi^{*}$ orbitals. The PS (the three thiothymines) all have $3 \pi$ and $3 \pi^{*}$ orbitals. Out of these only the 2 highest energy $\pi$ and 2 lowest energy $\pi^{*}$ orbitals were included to save computational cost. Further, we have confirmed that the chosen orbitals are involved in the transitions of the $T_{1}$ state in each respective molecule. The $6-31 \mathrm{G}^{* *}$ basis set was used for all calculations. All calculations were performed using the Molpro 2012 [41, 42] quantum chemical software package, and molecular orbitals were visualized using Molden [43]. The FCWD, diabatization of the nonadiabatic couplings, and rate constants were calculated using an in-house code.

\section{RESULTS AND DISCUSSION}

\section{A. Rate constants using MT-FGR and TD-FGR}

The PSs considered in this study include the family of thiothymines (Fig. 1), all of which have considerable singlet oxygen quantum yields. The starting energy and geometry for photosensitization corresponds to their respective $T_{1}$ minimum energy structure. Note that the bond length of $\mathrm{O}_{2}$ is always kept at its ground state minimum (1.21 $\mathrm{A}$ ). Based on previous studies which find 

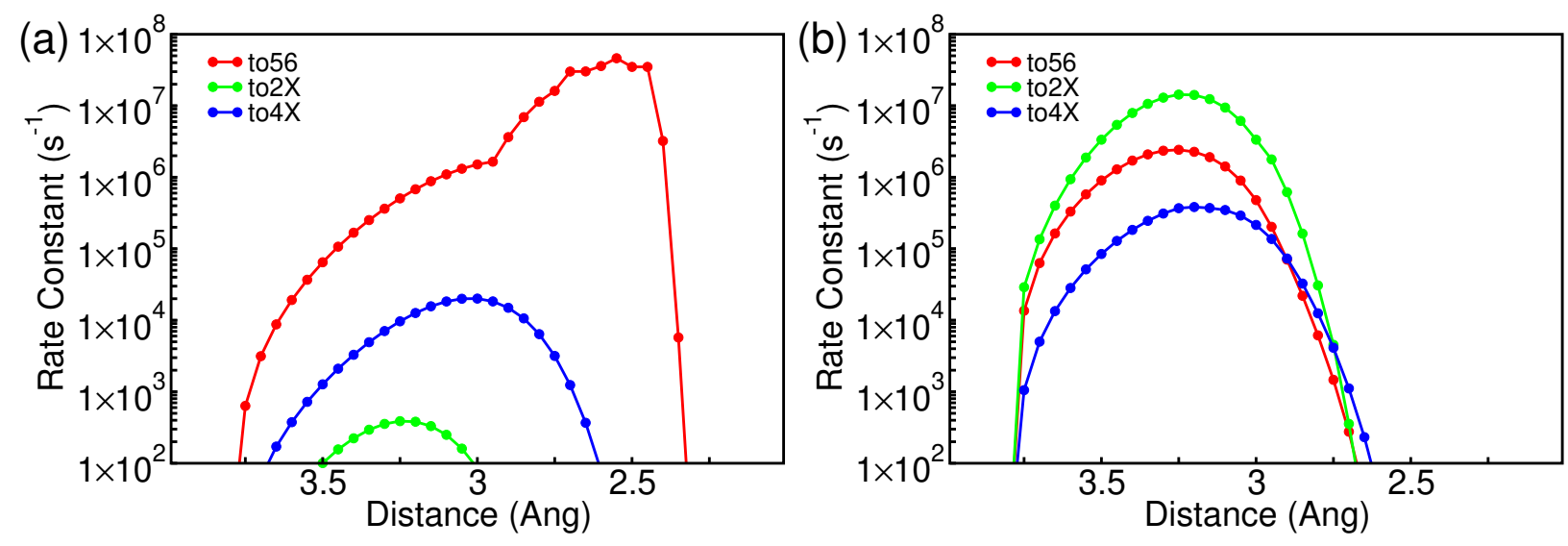

FIG. 4. Photosensitizing rate constants as a function of D calculated using MT-FGR for (a) $T_{1}^{\text {ring }}$ and (b) $T_{1}^{p y r}$ minima of $2 \mathrm{tThy}$.

one of the thiothymines, 2 tThy, to have a double well in its $T_{1}$ potential energy surface, we consider 2 triplet minima for this molecule (structures denoted as $T_{1}^{\text {ring }}$ and $T_{1}^{\text {pyr }}$ ) and the one relevant minimum for 4tThy and dtThy, which is the $T_{1}^{\text {ring }}$ structure (Fig. S3) [38, 44-46]. The main structural difference between $T_{1}^{\text {ring }}$ and $T_{1}^{\text {pyr }}$ is strong pyramidalization of the carbonyl or thionyl group at $\mathrm{C}^{2}$. For 4tThy and dtThy, the $T_{1}^{p y r}$ minimum is considerably high in energy and inaccessible, whereas a small energy difference exists between $T_{1}^{\text {ring }}$ and $T_{1}^{\text {pyr }}$ in $2 \mathrm{tThy}$, making both structures relevant $[38,47]$. As noted earlier, since ISC rates are fast [18] and triplet lifetimes are long [19, 20], ISC will not be a determining factor in calculating photosensitization rate constants.

The procedure to calculate photosensitization rate constants from the MT-FGR method, $k_{I F}^{M T}$ in Eq. 5, including the calculation of reorganization energies, $\lambda$, is outlined in detail in Reference [16]. These rate constants are shown in Fig. 4, for all three approach directions for both triplet minima of $2 \mathrm{tThy}$. However, rate constants for $4 \mathrm{tThy}$ and dtThy were calculated to be extremely low and the highest rate constants obtained for 4tThy and dtThy are $4.7 \times 10^{-3} \mathrm{~s}^{-1}$, and $4.2 \times$ $10^{-11} \mathrm{~s}^{-1}$, respectively, and along the to $4 \mathrm{X}$ direction for both molecules (Fig. S4).

The photosensitization rate constants, $k_{I F}^{T D}$, calculated from TD-FGR, are shown in Fig. 5. The highest rate constants for 2 tThy $T_{1}^{\text {ring }}, 2$ tThy $T_{1}^{p y r}$, 4 tThy and dtThy, are $8.53 \times 10^{8} s^{-1}, 8.64 \times$ $10^{4} s^{-1}, 1.29 \times 10^{7} s^{-1}$, and $3.08 \times 10^{7} s^{-1}$, respectively. Further, the orientation direction along which these maximum rate constants appear are also different: to56 for 2 tThy $T_{1}^{\text {ring }}$, to $2 \mathrm{X}$ for 2tThy $T_{1}^{p y r}$ and to $4 \mathrm{X}$ for both 4tThy and dtThy.

Experimentally, the rate constants at which molecular $\mathrm{O}_{2}$ quenches the $T_{1}$ state of 2tThy [19] and 4tThy [20] is found to be $(5.1 \pm 0.1) \times 10^{9} \mathrm{M}^{-1} \mathrm{~s}^{-1}$ and $(6.4 \pm 1.0) \times 10^{9} \mathrm{M}^{-1} \mathrm{~s}^{-1}$, respec- 

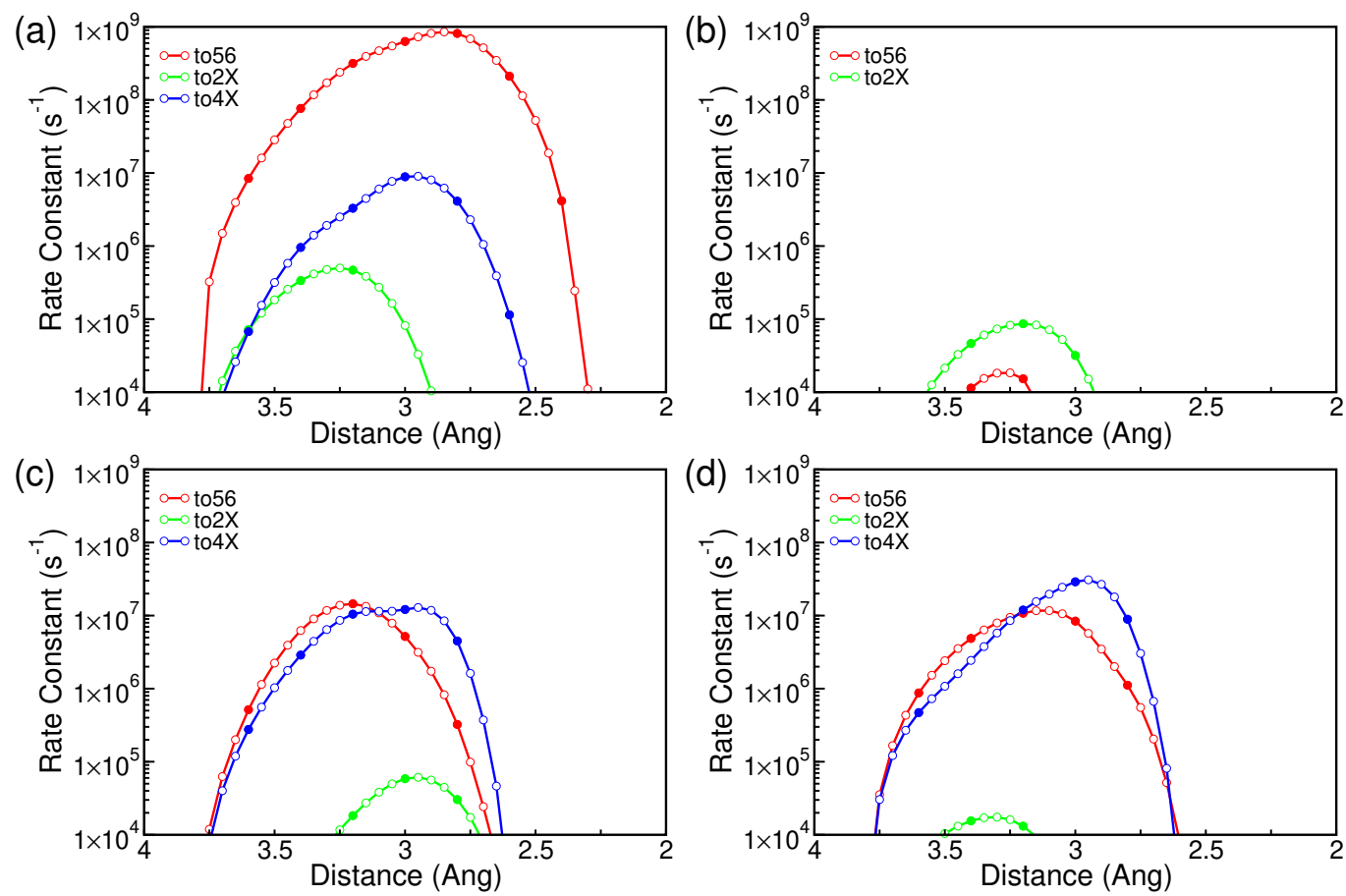

FIG. 5. Photosensitizing rate constants as a function of D calculated using TD-FGR for (a) $T_{1}^{\text {ring }}$ and (b) $T_{1}^{\text {pyr }}$ minima of $2 \mathrm{tThy}$, and $T_{1}^{\text {ring }}$ minimum of (c) 4tThy and (d) dtThy. Filled circles indicate values where FCWD is calculated explicitly and unfilled circles indicate values where FCWD is interpolated. Note that the diabatic coupling and Boltzmann factor are explicitly calculated for all points. Rates along to $4 \mathrm{X}$ in (b) are not seen as they are very low.

tively. These values can be thought of as a proxy for experimental photosensitization rate constants and are higher than those calculated in this work. Importantly, experiment suggests that both these molecules have similar photosensitization rates. Note however, that these experimental quenching rates are calculated in solvent (acetonitrile) at high concentrations of $\mathrm{O}_{2}$, while the rates modelled in this study are calculated between 1 PS and $1 \mathrm{O}_{2}$ molecule, in the gas phase.

We see that rate constants calculated using MT-FGR are severely underestimated for 4tThy and dtThy. On the other hand, rate constants calculated from TD-FGR for the $T_{1}^{\text {ring }}$ minimum of all three thiothymines are similar and correctly depict the trend in the experimental observations. Significantly, rate constants in 4tThy and dtThy differ by 9 and 17 orders of magnitude, respectively, between the two methods, clearly indicating the significance of quantum effects in photosensitization. While this acute difference between the two methods is surprising, rate constants estimated from Marcus theory are known to underestimate experimental values by 3-6 orders of magnitude [48]. In the forthcoming subsection we examine the reason for the large differences in calculated 
rate constants between MT-FGR and TD-FGR.

\section{B. Comparison of MT-FGR and TD-FGR}

Diabatic coupling, which is a key component in the rate constant calculation, is a common factor between both MT-FGR and TD-FGR (Eqs. 5 and 9) approaches. It increases as the PS and $\mathrm{O}_{2}$ come close and depends strongly on the overlap between the $\pi^{*}$ orbitals of $\mathrm{O}_{2}$ and the PS [15]. The diabatic couplings, as a function of D are shown in Fig. S5, and the orbitals which define the nature of the $T_{1}$ minimum of the thiothymines are shown in Fig. S3 and the relationship between the diabatic coupling and molecular overlap is clearly illustrated in all cases.

The $T_{1}^{\text {ring }}$ minimum in all 3 thiothymines have very little diabatic coupling along the to $2 \mathrm{X}$ direction and this can be directly correlated with the little to no electron density over the $\mathrm{C}^{2}=\mathrm{X}$ region in all these molecules. The only conformer to have significant electron density along this region ( $T_{1}^{p y r}$ of 2 tThy) is accordingly seen to have the highest diabatic coupling along the to $2 \mathrm{X}$ direction. Among the $T_{1}^{\text {ring }}$ minimum orbitals of all 3 thiothymines, 4 tThy and dtThy have significant electron density along the $C^{4}=S$ bond, while 2 tThy only has density along the $C^{5}=C^{6}$ bond. This is manifested in the highest diabatic coupling being present along the to $4 \mathrm{X}$ direction in 4tThy and dtThy and along the to56 direction in 2tThy. Overall, the highest coupling in 4tThy and dtThy (along to $4 \mathrm{X}$ ) is about 2 orders of magnitude higher than the highest coupling in 2 tThy (along to56), and this trend can also be tied back to the extent of orbital overlap. In 4tThy and dtThy, the $\pi^{*}$ orbitals of $\mathrm{O}_{2}$ overlap with $\pi^{*}$ orbitals which involve the larger $\mathrm{S}$ atom as opposed to the smaller $\mathrm{C}$ in $2 \mathrm{tThy}$. While one can argue that the $\mathrm{S}$ atom is involved in the orbitals of $T_{1}^{p y r}$ of 2tThy, and therefore the coupling in this case should be similar to the coupling values along the to $4 \mathrm{X}$ direction of $4 \mathrm{tThy}$ and dtThy, the low coupling is likely because of the $\mathrm{C}^{2}=\mathrm{S}$ bond being weaker due to strong pyramidalization and bond elongation, and hence, lower electron density. The remarkable role of diabatic coupling in photosensitization rate constants is evident when we see that the directions which have the highest diabatic coupling (to56 for $2 \mathrm{tThy} T_{1}^{\text {ring }}$, to $2 \mathrm{X}$ for $2 \mathrm{tThy} T_{1}^{p y r}$ and to $4 \mathrm{X}$ for both $4 \mathrm{tThy}$ and dtThy) are the same along which the calculated rate constant is the highest.

While the rate constant, $k_{I F}^{T D}$ in (Eq. 9), has a square dependence on the diabatic coupling, the Boltzmann factor can quench the rate with an exponential scaling. This is present in $k_{I F}^{M T}$ as well since $\Delta E^{\ddagger}$ in Eq. 5 (if entropy contributions are neglected) is composed of two terms: 
$\Delta E^{\ddagger}=\Delta E_{i}+E_{a}^{\ddagger}$, where $\Delta E_{i}$ is the same energy difference which is considered in the Boltzmann factor in Eq. 9, and $E_{a}^{\ddagger}$ is the energy barrier for $\mathrm{PS}-\mathrm{O}_{2}$ to reach the crossing between its reactant and product state (see Fig. 2a). Therefore, $\Delta E_{i}$ is also common for both rate constants, $k_{I F}^{M T}$ and $k_{I F}^{T D}$. The Boltzmann factor (with $\Delta E_{i}$ ) as a function of $\mathrm{D}$, along all 3 orientation directions, for all the thiothymines is shown in Fig. S6. The Boltzmann factor falls off faster in 4tThy and dtThy as compared to $2 \mathrm{tThy}$. Paradoxically, this is for the same reason that the diabatic coupling in $4 \mathrm{tThy}$ and dtThy was higher than in 2tThy: as the $\mathrm{O}_{2}$ approaches a double bond which involves the $\mathrm{S}$ atom, the greater orbital overlap enhances diabatic coupling while the steric hindrance is also greater due to the larger size of $\mathrm{S}$ (as compared to $\mathrm{O}$ ).

The key difference between $k_{I F}^{M T}$ and $k_{I F}^{T D}$ is that the former has a negative exponential dependence on $E_{a}^{\ddagger}$, while the latter is proportional to FCWD. The FCWD values as a function of D for all three thiothymines are found to fall within the same range (see Fig. S7). $E_{a}^{\ddagger}$, however, is very different among the thiothymines. The calculated $E_{a}^{\ddagger}$ in 2 thy (both conformers) is about $0.2 \mathrm{eV}$, while that in $4 \mathrm{tThy}$ and dtThy is about $0.8 \mathrm{eV}$ and $1.1 \mathrm{eV}$, respectively. Note that $E_{a}^{\ddagger}$ depends on $\mathrm{D}$, and these values are for a $\mathrm{D}$ when the diabatic coupling is significant and $\Delta E_{i}$ is still small. The high barrier in 4tThy and dtThy essentially extinguishes any rate that may arise because of the diabatic coupling. This is due to the inherent classical interpretation of MT-FGR. Unlike a quantum process, where the state transfer can take place at any geometry, not just the crossing, within the classical regime, state transfer can take place only after the crossing is reached.

The MT-FGR approach works well when $k_{B} T<<\hbar \omega$ for all vibrational frequencies [16, 24]. While modeling photosensitization, it is adequate that the mode that is responsible for the deactivation of the isolated PS satisfy this condition, i.e., the mode along which the $T_{1}$ minimum moves to reach the MECP with the ground state, to be less than $200 \mathrm{~cm}^{-1}$ (this values corresponds to $\sim 1 k_{B} T$ at $298 \mathrm{~K}$ ) [16]. This is essentially the mode along which the TT0 surface of the PS-O 2 composite system crosses with the SS2 state. Therefore, we have evaluated if MT-FGR is a suitable approach for the thiothymines by calculating the frequency of the mode which is responsible for deactivation of the PS from its $T_{1}$ minimum to its MECP with the ground state. This has been done by expressing the difference in geometries between the MECP and $T_{1}$ minimum structures in terms of the normal modes of the $T_{1}$ minimum. Finally, the total resultant frequency of the mode is calculated as a linear combination of the frequency of each mode multiplied by the contribution of that mode. This procedure has been followed since the geometry changes between the MECP and $T_{1}$ minimum were too diverse to be assigned to any one normal mode of the $T_{1}$ minimum through 
visual inspection. These resultant frequencies for $2 \mathrm{tThy}\left(T_{1}^{\text {ring }}\right), 2 \mathrm{tThy}\left(T_{1}^{\text {pyr }}\right), 4 \mathrm{t}$ Thy $\left(T_{1}^{\text {ring }}\right)$ and dtThy $\left(T_{1}^{\text {ring }}\right)$ were calculated to be $199 \mathrm{~cm}^{-1}, 141 \mathrm{~cm}^{-1}, 379 \mathrm{~cm}^{-1}$ and $358 \mathrm{~cm}^{-1}$, respectively. Since the frequency of the deactivating mode is required to be less than $200 \mathrm{~cm}^{-1}$, the calculated values suggest that MT-FGR is unsuitable for 4tThy and dtThy. This high frequency of this mode can also be tied back to high $E_{a}^{\dagger}$ values. Consequently, the classical treatment in MT-FGR is inadequate and suggests the need for a quantum approach.

\section{v. CONCLUSIONS}

In this study we have developed a general approach for calculating the rate of photosensitization of oxygen to give singlet oxygen by a PS. We have obtained mechanistic insights into photosensitization, and in particular, the importance of quantum effects. We have calculated photosensitization rate constants for thiothymines as the PSs using two approaches: MT-FGR and TD-FGR. MT-FGR is the classical limit of FGR, and its implementation for bimolecular systems was proposed by Bai and Barbatti [16]. While TD-FGR retains the quantum nature of FGR, it has so far only been used for unimolecular photophysical reactions like IC and ISC. In this study, we have adapted TD-FGR for bimolecular reactions. We describe the complexities that arise in the case of bimolecular systems and how to circumvent them. Using TD-FGR treats the reaction quantum mechanically and allows rigorous treatment of FCWD.

Relative rate constants obtained from TD-FGR are in reasonable agreement with experimental results, while those from MT-FGR are severely underestimated for 4tThy and dtThy by several orders of magnitude. It is worth noting that the MT-FGR formalism fails for 4tThy and dtThy because the mode responsible for triplet deactivation in these molecules has a very high frequency, and MT-FGR is only valid if the opposite is true. This high frequency also translates to a high energy barrier for ISC in 4tThy and dtThy $(\sim 0.8-1.1 \mathrm{eV})$. Essentially, the physical interpretation of MT-FGR requires the PS- $\mathrm{O}_{2}$ complex to surmount the barrier to reach the crossing, while TD-FGR allows the formation of the product if the diabatic coupling is sufficiently high.

It is interesting that $2 \mathrm{tThy}$ and $4 \mathrm{tThy}$, two very similar molecules, have such different electronic properties so as to warrant different approaches to calculating the rate at which they photosensitize $\mathrm{O}_{2}$. Also, 4tThy and dtThy are similar to each other, while being different to $2 \mathrm{t}$ thy, pointing to a position-dependent effect at play [38, 47].

In conclusion, we have adapted the TD-FGR approach for bimolecular reactions, and the suc- 
cess of this method with all three thiothymines, while MT-FGR fails for 4tThy and dtThy, demonstrates the generality of this approach. More importantly, this study finds that a method to calculate photosensitization rate constants, must necessarily describe the inherent quantum effects that drive the process.

\section{ACKNOWLEDGMENTS}

For financial support, we acknowledge the Science and Engineering Research Board, Government of India (Project No. GAP/DST-SERB/CHE-12-0086). We also acknowledge C-DAC Pune for computing facilities. M.A.M. thanks IISER Pune for a research fellowship. The authors thank Avdhoot Datar and Satyam Ravi for useful discussions.

[1] J. D. Nguyen, E. M. D'amato, J. M. Narayanam, and C. R. Stephenson, Nature Chemistry 4, 854 (2012).

[2] J. W. Tucker and C. R. Stephenson, The Journal of organic chemistry 77, 1617 (2012).

[3] S. Fukuzumi and K. Ohkubo, Chemical Science 4, 561 (2013).

[4] J. Xuan and W.-J. Xiao, Angewandte Chemie International Edition 51, 6828 (2012).

[5] D. Ravelli, M. Fagnoni, and A. Albini, Chemical Society Reviews 42, 97 (2013).

[6] Y. Y. Cheng, B. Fückel, R. W. MacQueen, T. Khoury, R. G. Clady, T. F. Schulze, N. Ekins-Daukes, M. J. Crossley, B. Stannowski, K. Lips, et al., Energy and Environmental Science 5, 6953 (2012).

[7] B. W. Henderson and T. J. Dougherty, Photochemistry and photobiology 55, 145 (1992).

[8] T. J. Dougherty, C. J. Gomer, B. W. Henderson, G. Jori, D. Kessel, M. Korbelik, J. Moan, and Q. Peng, JNCI: Journal of the national cancer institute 90, 889 (1998).

[9] J. Zhao, W. Wu, J. Sun, and S. Guo, Chemical Society Reviews 42, 5323 (2013).

[10] L. Shen and H.-F. Ji, Journal of Photochemistry and Photobiology B: Biology 91, 96 (2008).

[11] E. L. Coitiño, A. Mella, and G. I. Cárdenas-Jirón, Journal of Photochemistry and Photobiology A: Chemistry 294, 68 (2014).

[12] C. Espinoza, A. Trigos, and M. E. Medina, The Journal of Physical Chemistry A 120, 6103 (2016).

[13] L. Martínez-Fernández, G. Granucci, M. Pollum, C. E. Crespo-Hernández, M. Persico, and I. Corral, Chemistry-A European Journal 23, 2619 (2017). 
[14] E. Dumont, M. Wibowo, D. Roca-Sanjuán, M. Garavelli, X. Assfeld, and A. Monari, The journal of physical chemistry letters 6, 576 (2015).

[15] J. J. Serrano-Pérez, G. Olaso-Gonzalez, M. Merchan, and L. Serrano-Andres, Chemical Physics 360, 85 (2009).

[16] S. Bai and M. Barbatti, Journal of chemical theory and computation 13, 5528 (2017).

[17] S. Bai and M. Barbatti, The journal of physical chemistry letters 8, 5456 (2017).

[18] M. Pollum, S. Jockusch, and C. E. Crespo-Hernández, J. Am. Chem. Soc. 136, 17930 (2014), pMID: 25506742, http://dx.doi.org/10.1021/ja510611j.

[19] H. Kuramochi, T. Kobayashi, T. Suzuki, and T. Ichimura, J. Phys. Chem. B 114, 8782 (2010), pMID: 20552955.

[20] Y. Harada, T. Suzuki, T. Ichimura, and Y.-Z. Xu, J. Phys. Chem. B 111, 5518 (2007), pMID: 17439266.

[21] P. A. M. Dirac, Proceedings of the Royal Society of London. Series A, Containing Papers of a Mathematical and Physical Character 114, 243 (1927).

[22] C. Cohen-Tannoudji, B. Diu, and F. Laloè, Quantum mechanics. 2, Textbook physics (John Wiley and Sons, 1977).

[23] J. Tatchen, N. Gilka, and C. M. Marian, Physical chemistry chemical physics 9, 5209 (2007).

[24] M. Bixon and J. Jortner, Advances in Chemical Physics 106, 35 (1999).

[25] M. Etinski, J. Tatchen, and C. M. Marian, The Journal of chemical physics 134, 154105 (2011).

[26] R. Borrelli and A. Peluso, Physical Chemistry Chemical Physics 13, 4420 (2011).

[27] R. Borrelli, A. Capobianco, and A. Peluso, Chemical Physics 440, 25 (2014).

[28] T. J. Penfold, E. Gindensperger, C. Daniel, and C. M. Marian, Chemical reviews 118, 6975 (2018).

[29] Y. Niu, Q. Peng, C. Deng, X. Gao, and Z. Shuai, The Journal of Physical Chemistry A 114, 7817 (2010).

[30] M. Hayashi, A. Mebel, K. Liang, and S. Lin, The Journal of chemical physics 108, 2044 (1998).

[31] R. Islampour and M. Miralinaghi, The Journal of Physical Chemistry A 111, 9454 (2007).

[32] Q. Peng, Y. Yi, Z. Shuai, and J. Shao, The Journal of chemical physics 126, 114302 (2007).

[33] F. G. Mehler, Journal für die reine und angewandte Mathematik 66, 161 (1866).

[34] F. Duschinsky, Acta Physicochim. URSS 7, 551 (1937).

[35] C. Eckart, Physical Review 47, 552 (1935).

[36] S. V. Krasnoshchekov, E. V. Isayeva, and N. F. Stepanov, The Journal of Chemical Physics 140, 
154104 (2014).

[37] M. Baer, Beyond Born-Oppenheimer: electronic nonadiabatic coupling terms and conical intersections (John Wiley \& Sons, 2006).

[38] M. A. Manae and A. Hazra, The Journal of Physical Chemistry A 123, 10862 (2019).

[39] P. J. Knowles and H.-J. Werner, Chemical physics letters 115, 259 (1985).

[40] H.-J. Werner and P. J. Knowles, The Journal of chemical physics 82, 5053 (1985).

[41] H.-J. Werner, P. J. Knowles, G. Knizia, F. R. Manby, and M. Schütz, Wiley Interdisciplinary Reviews: Computational Molecular Science 2, 242 (2012).

[42] H.-J. Werner, P. Knowles, G. Knizia, F. Manby, M. Schütz, P. Celani, T. Korona, R. Lindh, A. Mitrushenkov, G. Rauhut, et al., See http://www. molpro. net (2012).

[43] G. Schaftenaar and J. H. Noordik, Journal of computer-aided molecular design 14, 123 (2000).

[44] S. Bai and M. Barbatti, Physical Chemistry Chemical Physics 19, 12674 (2017).

[45] D. Koyama, M. J. Milner, and A. J. Orr-Ewing, The Journal of Physical Chemistry B 121, 9274 (2017).

[46] J. A. Sánchez-Rodríguez, A. Mohamadzade, S. Mai, B. Ashwood, M. Pollum, P. Marquetand, L. González, C. E. Crespo-Hernández, and S. Ullrich, Physical Chemistry Chemical Physics 19, 19756 (2017).

[47] M. A. Manae and A. Hazra, The Journal of Physical Chemistry A 121, 8147 (2017).

[48] J. P. Martínez, D. E. Trujillo-Gonzalez, A. W. Gotz, F. L. Castillo-Alvarado, and J. I. Rodriguez, The Journal of Physical Chemistry C 121, 20134 (2017) 